\title{
Has the quality of serosurveillance in low- and middle-income countries improved since the last HIV estimates round in 2007? Status and trends through 2009
}

\author{
Jesus M Garcia Calleja, ${ }^{1}$ J Jacobson, ${ }^{1}$ R Garg, ${ }^{1}$ N Thuy, ${ }^{1}$ A Stengaard, ${ }^{1}$ M Alonso, ${ }^{1}$ \\ H O Ziady, ${ }^{1}$ L Mukenge, ${ }^{1}$ S Ntabangana, ${ }^{1} \mathrm{D}$ Chamla, ${ }^{1}$ A Alisalad, ${ }^{1}$ E Gouws, ${ }^{2}$ \\ K Sabin, ${ }^{1}$ Y Souteyrand ${ }^{1}$
}

${ }^{1}$ World Health Organization, Geneva, Switzerland ${ }^{2}$ WHO/HIV department, UNAIDS, Geneva, Switzerland

\section{Correspondence to}

Dr Jesus M García Calleja, WHO, 20, Avenue Appia, Geneva 27, Switzerland; callejaj@who.int

Accepted 7 October 2010

\begin{abstract}
Background HIV surveillance systems aim to monitor trends of HIV infection, the geographical distribution and its magnitude, and the impact of HIV. The quality of HIV surveillance is a key element in determining the uncertainty ranges around HIV estimates. This paper aims to assess the quality of HIV surveillance systems in low- and middleincome countries in 2009 compared with 2007.

Methods Four dimensions related to the quality of surveillance systems are assessed: frequency and timeliness of data; appropriateness of populations; consistency of locations and groups; and representativeness of the groups. An algorithm for scoring the quality of surveillance systems was used separately for low and concentrated epidemics and for generalised epidemics.
\end{abstract}

Results The number of countries categorised as fully functioning in 2009 was 35, down from 40 in 2007. 47 countries were identified as partially functioning, while 56 were categorised as poorly functioning. When compared with 2007, the quality of HIV surveillance remains similar. The number of ANC sites in sub-Saharan Africa has increased over time. The number of countries with low and concentrated epidemics that do not have functioning HIV surveillance systems has increased from 53 to 56 between 2007 and 2009.

Conclusion Overall, the quality of surveillance in low- and middle-income countries has remained stable. Still too many countries have poorly functioning surveillance systems. Several countries with generalised epidemics have conducted more than one populationbased survey which can be used to confirm trends. In countries with concentrated or low-level epidemics, the lack of data on high-risk populations remains a challenge.

\section{INTRODUCTION}

The main goal of an HIV surveillance system is to monitor trends over time in HIV prevalence, incidence, mortality and behaviours associated with HIV transmission, as well as to assess the population level burden of HIV and the geographical distribution. Furthermore, data produced by HIV surveillance systems are essential for assessing the economic, health and demographic impacts of the epidemic, for monitoring the impact of intervention efforts and for predicting future epidemic trends.

The UNAIDS/WHO Working Group on Global HIV/AIDS and STI Surveillance regularly publishes guidelines for conducting HIV surveillance. ${ }^{1}$ The last HIV sentinel surveillance guidelines in 2003 recommend that countries with generalised epidemics continue to implement HIV sentinel surveillance among antenatal clinic (ANC) attendees and to supplement these with nationally representative population-based behavioural surveys which include HIV testing. In countries with low-level and concentrated epidemics, it is recommended to use HIV case reporting, despite possible duplication or under-reporting, and Integrated Biological Behaviour Surveillance Surveys (IBBSS) among populations with high-risk behaviours. Guidelines are available from the UNAIDS and WHO websites (http://www.unaids. org/en/KnowledgeCentre/HIVData/Epidemiology/ epiworkinggrp. and http://www.who.int/hiv/pub/ surveillance/en/). Epidemic definitions are provided in the Methods and data sources section. The data collected from these surveillance systems are used on an ongoing basis by various countries, with the support of UNAIDS and WHO, to produce national estimates of HIV prevalence and impact, including HIV incidence, AIDS mortality and treatment needs.

A method for assessing the quality of national surveillance systems was developed by WHO and UNAIDS in 2000 and was applied in 2002, 2005 and $2008 .^{2-4}$ This was done utilising HIV surveillance data available from the United States Census Bureau's HIV surveillance database, European Centre for Disease Control (ECDC prevalence database and country reports on HIV prevalence and surveillance activities available at the time to WHO and UNAIDS).

The main objective of this paper is to report on changes in the quality of HIV surveillance systems between 2007 and 2009. In addition, we examine changes over time in the number of ANC sentinel sites in sub-Saharan Africa and national population surveys conducted between 2000 and 2009 as the pillars of surveillance in countries most affected by the epidemic. Finally, we identify gaps in the data needed to guide national AIDS programmes in making policy decisions.

\section{METHODS AND DATA SOURCES}

Data on HIV surveillance in countries were collected from different sources for the period 2001 
to 2009. In addition to the United States Census Bureau's HIV surveillance database (http://www.census.gov/ipc/www/ hivaidsn.html), WHO regional offices and country staff provided information on the number and location of surveillance activities in their countries using a standardised template. The template was sent to WHO Regional offices, so they could collect the information in a standard manner based on an Excel file with the different scoring parameters. Efforts were made on the collection of data in the most recent years, 2005-2008. All the strategic information focal points that work in WHO regional offices returned the templates providing data gathered at country level. There were no other searchs of published articles or reviews in journals.

\section{EPIDEMIC CLASSIFICATION}

National HIV epidemics were classified as low-level, concentrated or generalised according to standard definitions first introduced in 2000. ${ }^{1}$ Countries with low-level epidemics are those in which HIV transmission occurs mostly among most at-risk populations where HIV prevalence has not consistently exceeded $5 \%$ in any subpopulation. An epidemic is referred to as concentrated if the estimated HIV prevalence is consistently over $5 \%$ in at least one subpopulation and below $1 \%$ in the general adult population (age 15-49 years) in urban areas. In countries with generalised epidemics, HIV prevalence is firmly established in the general adult population (prevalence $\geq 1 \%$ ).

\section{Scoring the quality of surveillance systems for generalised epidemics}

Previous assessments of the quality of HIV surveillance systems relied on coding schemes that summarise four quality dimensions as developed by Walker et $a l^{2}$ and updated by Lyerla et al in $2008 .^{4}$ Similar dimensions to those used by Lyerla et al ${ }^{4}$ were used to allow for comparison between 2007 and 2009. The only difference is that the years being revised were increased (2001 to 2009) to include the most recent surveillance data: frequency and timeliness of data collection; appropriateness of populations under surveillance; consistency of the sites/location and groups measured over time; and coverage/representativeness of the groups for the adult populations. Scores were computed for each of the quality dimensions and then combined to create an overall score of the quality of the surveillance system for each country included in this process.

\section{Frequency and timeliness}

Frequency and timeliness were measured as the number of times a national sentinel surveillance study or a national populationbased HIV prevalence survey had been conducted between 2001 and 2009 (range 0 to a maximum of 9). As was done earlier by Lyerla et al, ${ }^{4}$ countries where data had been collected in the last 2 years were given an additional 1 for timeliness; others were given a 0 . The sum of these two variables was used as the overall measure of frequency and timeliness (maximum frequency and timeliness score of 10).

\section{Appropriateness}

An appropriate surveillance system was defined as one in which data had been collected in the last 9 years in urban as well as rural sites from antenatal clinic surveillance. In this round of assessment, if a national population-based survey had been conducted between 2001 and 2009, it was also counted as 'appropriate' Countries with an appropriate system were scored as 1; all others were scored as zero.

\section{Consistency}

Scoring for consistency was a judgement made by the reviewer of the country data to reflect the degree to which surveillance activities permitted a country to accurately assess epidemic trends in the same populations and locations over time. The score for consistency was made on a three-point scale, with zero representing no pattern of consistency in urban and rural sites from antenatal clinic surveillance; a score of 1 was given when there was some repetition in sites, providing some information about trends; and a score of 2 was given for surveillance systems where a clear pattern of consistency was evident. If countries had more than one national population survey, two extra points were scored (maximum consistency score of 5).

\section{Coverage}

Coverage was scored similarly to the 2007 round of assessment. It was scored on a four-point scale, $0-3$, with 0 reflecting poor coverage, 1 reflecting some evidence of an increasing surveillance capacity, 2 if the surveillance system in antenatal clinics is fully representational in urban and rural areas, and 3 if a DHS or national population-based survey with HIV testing was conducted in addition to sentinel surveillance.

The number of ANC sites in the most affected region, subSaharan Africa, was also examined as they are part of a regular surveillance system. A simple count of sentinel sites for ANC was done looking at the reports available. No distinction was made for rural or urban sites.

\section{Overall quality of the surveillance systems in generalised epidemics}

As in the last revision of quality of HIV surveillance systems, ${ }^{4}$ the same combinations of scores were used, but 2 years were added. The overall quality of the surveillance system was determined by the combination of the scores for each of the four dimensions. The sum of these four dimensions ranged from 0 to 19, with frequency and timeliness contributing about half of the total. As in the past, three categories of quality were used: fully implemented, partially implemented and poorly implemented. The distribution of country scores was reviewed to determine categories of quality. Countries scoring greater than 12 were determined to be fully functioning, representing surveillance systems that were timely, frequent, appropriate and representative. Countries scoring 7 to 11 were rated as partially implemented, characterised by having some features of a highquality system, but not all. Many of these countries needed more frequent surveillance. Countries scoring lower than 7 were characterised as poorly functioning in which none of the countries were determined to have sufficient data to track the trends in the epidemic. As a result of this analysis, gaps in surveillance systems can be identified and utilised to enhance country-specific surveillance systems.

\section{Scoring surveillance quality in countries with concentrated and low-level epidemics}

In countries with low-level or concentrated epidemics, surveillance quality was assessed differently, as the risk of infection is concentrated in population groups that report behaviours associated with HIV infection. Consequently, HIV surveillance data to monitor trends should come from these groups at a higher risk of infection.

Surveillance systems in these countries were assessed based on the presence of data from four of the groups typically most at risk for infection during the period 2001 to 2009: commercial sex 
workers (CSW), clients of CSW, men who have sex with men (MSM) and injecting-drug users (IDUs). Data were also collected on surveillance conducted among patients seeking care for sexually transmitted infections, which can serve as a proxy for high-risk individuals in some settings. For each population, the years in which data were collected were also recorded.

As for countries with generalised epidemics, the quality score for countries with low-level or concentrated epidemics was based on the four dimensions outlined above. Frequency was scored as described above, as was timeliness, with a maximum score of 10 . However, as surveillance recommendations for such epidemics do not call for complete Geographic coverage-since populations are often clustered in urban areas-the appropriateness and coverage dimensions were combined and defined by the number of populations under surveillance. For low-level epidemics, the highest score 3 was assigned to countries collecting data from all groups at high risk for infection. For countries with concentrated epidemics, surveillance needed to include high-risk groups as well as pregnant women in urban areas. If countries collect data in two high risk groups would received a score of 2 , but if they collected only information from one high risk group, they will receive a score of 1.

As initially proposed by Walker et $a l^{2}$ consistency was again judged by reviewing the sites over time. For consistency, the same sites over different periods of time were included. Three points were given, two for some consistency and one for no or very little consistent use of sites. Consistency is defined as having a minimum of three data points.

\section{Overall quality of the sentinel surveillance systems: concentrated and low-level epidemics}

As in the case of countries with generalised epidemics, a summary score was developed by combining different dimensions of surveillance. However, for concentrated and lowlevel epidemics, the scores could range from 0 to 17 . Countries were ranked by total scores, and cut-off scores for determining the overall quality were based on score distributions. For both concentrated and low-level epidemics, poorly functioning surveillance systems received a score of 0 to 4 , partially functioning systems were scored 5 to 11 , and fully functioning systems were scored 12 or higher.

\section{RESULTS}

Results of the quality of HIV surveillance in 138 low and middleincome countries are presented in table 1 by geographic region and type of epidemic. Forty-one countries are categorised as having generalised epidemics, 51 countries have concentrated epidemics, and 45 countries in this assessment have a low-level HIV epidemic based on the data available in 2009.

Overall, 35 countries have a fully functioning system, while 46 have partially functioning systems, and the rest, 57, have low-quality surveillance systems.

When these overall results are compared with 2007, seven countries have been downgraded from fully implementing systems to partially functioning systems (Ukraine, Mexico, Angola, Côte d'Ivoire, Mozambique, Namibia, Swaziland), and three have been upgraded to fully functioning systems (Gambia, Liberia and Senegal). However, overall the number of countries in the two lower categories remains similar, as presented in table 2 .

Among the 45 countries scored in sub-Saharan Africa, 39 are categorised as having generalised epidemics. Twenty-one countries in this region have systems that would be categorised as fully functioning, 15 as partially functioning and nine as not functioning. Many of the countries in the region have conducted national population-based surveys in which HIV testing has been included, which are often used to adjust national HIV prevalence estimates based on ANC sentinel surveillance data. Moreover, countries such as Kenya and South Africa have conducted three national population survey since 2002, while others have already conducted two (Mali, Zambia, Niger, Tanzania and Burundi). On the other hand, nine countries do not have surveillance systems that could be characterised as even partially functioning. These countries do not have basic surveillance activities that will allow for tracking the epidemic.

When comparing these results with the 2007 assessment by Lyerla et $a l^{4}$ three countries in this region have been downgraded from fully functioning systems (Angola, Côte d'Ivoire, Mozambique) to partially functioning, and the number of poorly functioning systems has increased by two countries (Djibouti, Equatorial Guinea), while the number of countries with partially systems has decreased by four countries, as shown in table 3.

In the Southern African subregion, which represents more than $50 \%$ of the total HIV burden in Africa, most countries have fully functioning surveillance systems, with the exception of Namibia, Mozambique and Swaziland, which were downgraded from fully to partially functioning because of the lack of consistency of data available in the last 2 years.

Declines in the quality score were mostly due to a lack of consistency and difficulty in assessing trends. For instance, efforts to improve surveillance systems in Central Africa were recorded in 2004-2006 but declined in 2007-2008. In this subregion, most of the countries have only partially functioning systems, despite conducting national population surveys in the mid-2000s. West Africa and the Horn of Africa also experienced declines in surveillance quality overall.

In other subregions, the quality has remained very similar, with no major changes between 2007 and 2009, as presented in table 3 .

\section{EXTENSION OF DATA SOURCES ANC sentinel surveillance sites}

For sub-Saharan Africa, we assessed the total number of sites included in HIV sentinel surveillance in countries, as presented in figure 1. Overall, more than 4000 sentinel ANC sites were included in surveillance efforts in countries in 2007-2008, which is a considerable increase over the 2200 reported in 2005-2006. Much of this increase can be attributed to one country, South Africa, where the number of sentinel sites increased from 457 in 2006 to 1427 in $2008 .^{6}$ Still, even when excluding South Africa from the analysis, there has been a steady increase in the number of sites, since HIV sentinel surveillance systems were recommended in 1991 by $\mathrm{WHO} / \mathrm{GPA}^{7}$ as a source for tracking epidemic trends.

\section{National population-based surveys}

National population-based surveys with HIV testing have been conducted in an increasing number of countries since 2001. Since then, 38 countries globally have conducted one or more surveys, and nine have conducted more than one, as shown in table 4. Several other countries are currently implementing these surveys in 2009 and 2010, and others are planning to conduct surveys in the next 5 years (http://www.measuredhs.com/aboutdhs/).

National population-based surveys have also been conducted in some countries outside Africa, including Cambodia, Papua New Guinea and one province in Vietnam, and twice in 
Table 1 Classification of the epidemic and estimated quality of surveillance by country, 2009

\begin{tabular}{|c|c|c|}
\hline Country/region & State of epidemic & Quality rating 2009 \\
\hline \multicolumn{3}{|l|}{ Caribbean } \\
\hline Bahamas & C & 2 \\
\hline Barbados & C & 1 \\
\hline Cuba & C & 1 \\
\hline Dominican Republic & $\mathrm{C}$ & 3 \\
\hline Haiti & G & 3 \\
\hline Jamaica & $\mathrm{C}$ & 2 \\
\hline Trinidad and Tobago & $\mathrm{C}$ & 1 \\
\hline \multicolumn{3}{|l|}{ East Asia and Pacific } \\
\hline China & $\mathrm{C}$ & 3 \\
\hline Fiji & $\mathrm{L}$ & 1 \\
\hline Mongolia & $\mathrm{L}$ & 2 \\
\hline Papua New Guinea & C & 2 \\
\hline \multicolumn{3}{|c|}{ Eastern Europe and Central Asia } \\
\hline Armenia & C & 1 \\
\hline Azerbaijan & $\mathrm{L}$ & 2 \\
\hline Belarus & C & 2 \\
\hline Bosnia and Herzegovina & $\mathrm{L}$ & 1 \\
\hline Bulgaria & $\mathrm{L}$ & 1 \\
\hline Croatia & $\mathrm{L}$ & 1 \\
\hline Czech Republic & $\mathrm{L}$ & 2 \\
\hline Estonia & $\mathrm{L}$ & 2 \\
\hline Georgia & $\mathrm{L}$ & 2 \\
\hline Hungary & $\mathrm{L}$ & 1 \\
\hline Kazakhstan & $\mathrm{C}$ & 1 \\
\hline Kyrgyzstan & $\mathrm{L}$ & 2 \\
\hline Latvia & C & 2 \\
\hline Lithuania & $\mathrm{L}$ & 1 \\
\hline Poland & C & 1 \\
\hline Republic of Moldova & C & 1 \\
\hline Romania & $\mathrm{L}$ & 2 \\
\hline Russian Federation & C & 2 \\
\hline Slovakia & $\mathrm{L}$ & 1 \\
\hline Tajikistan & $\mathrm{L}$ & 1 \\
\hline Turkmenistan & $\mathrm{L}$ & 1 \\
\hline Ukraine & $\mathrm{C}$ & 2 \\
\hline Uzbekistan & $\mathrm{C}$ & 1 \\
\hline \multicolumn{3}{|l|}{ Latin America } \\
\hline Argentina & $\mathrm{C}$ & 3 \\
\hline Belize & $\mathrm{C}$ & 1 \\
\hline Bolivia & $\mathrm{L}$ & 1 \\
\hline Brazil & C & 3 \\
\hline Chile & C & 1 \\
\hline Colombia & C & 2 \\
\hline Costa Rica & C & 1 \\
\hline Ecuador & C & 2 \\
\hline El Salvador & C & 2 \\
\hline Guatemala & C & 2 \\
\hline Guyana & C & 2 \\
\hline Honduras & C & 2 \\
\hline Mexico & C & 2 \\
\hline Nicaragua & C & 1 \\
\hline Panama & C & 1 \\
\hline Paraguay & C & 2 \\
\hline Peru & C & 3 \\
\hline Suriname & C & 2 \\
\hline Uruguay & C & 2 \\
\hline Venezuela & C & 1 \\
\hline \multicolumn{3}{|l|}{ North Africa and Middle East } \\
\hline Algeria & $\mathrm{L}$ & 1 \\
\hline Bahrain & $\mathrm{L}$ & 1 \\
\hline Cyprus & $\mathrm{L}$ & 1 \\
\hline
\end{tabular}

Table 1 Continued

\begin{tabular}{|c|c|c|}
\hline Country/region & State of epidemic & Quality rating 2009 \\
\hline Egypt & L & 1 \\
\hline Iraq & L & 1 \\
\hline Israel & C & 1 \\
\hline Jordan & L & 1 \\
\hline Kuwait & L & 1 \\
\hline Lebanon & L & 1 \\
\hline Libyan Arab Jamahiriya & L & 1 \\
\hline Morocco & L & 2 \\
\hline Oman & L & 1 \\
\hline Qatar & L & 1 \\
\hline Saudi Arabia & L & 1 \\
\hline Sudan & G & 2 \\
\hline Syrian Arab Republic & L & 1 \\
\hline Tunisia & L & 1 \\
\hline Turkey & L & 1 \\
\hline United Arab Emirates & L & 1 \\
\hline Yemen & L & 1 \\
\hline \multicolumn{3}{|l|}{ South and South-East Asia } \\
\hline Afghanistan & L & 1 \\
\hline Bangladesh & L & 3 \\
\hline Bhutan & L & 1 \\
\hline Brunei Darussalam & L & 1 \\
\hline Cambodia & C & 3 \\
\hline India & C & 3 \\
\hline Indonesia & C & 2 \\
\hline Iran (Islamic Republic of) & L & 2 \\
\hline Lao People's Democratic Republic & L & 2 \\
\hline Malaysia & C & 2 \\
\hline Maldives & L & 1 \\
\hline Myanmar & C & 3 \\
\hline Nepal & C & 3 \\
\hline Pakistan & L & 2 \\
\hline Philippines & L & 2 \\
\hline Singapore & C & 1 \\
\hline Sri Lanka & L & 2 \\
\hline Thailand & C & 3 \\
\hline Viet Nam & $\mathrm{C}$ & 3 \\
\hline \multicolumn{3}{|l|}{ Sub-Saharan Africa } \\
\hline Angola & G & 2 \\
\hline Benin & G & 3 \\
\hline Botswana & G & 3 \\
\hline Burkina Faso & G & 3 \\
\hline Burundi & G & 3 \\
\hline Cameroon & G & 2 \\
\hline Central African Republic & G & 2 \\
\hline Chad & G & 2 \\
\hline Comores & C & 1 \\
\hline Congo & G & 2 \\
\hline Côte d'Ivoire & G & 2 \\
\hline Democratic Republic of Congo & G & 2 \\
\hline Djibouti & G & 1 \\
\hline Equatorial Guinea & G & 1 \\
\hline Eritrea & G & 2 \\
\hline Ethiopia & G & 3 \\
\hline Gabon & G & 1 \\
\hline Gambia & G & 3 \\
\hline Ghana & $\mathrm{G}$ & 3 \\
\hline Guinea & G & 1 \\
\hline Guinea-Bissau & G & 1 \\
\hline Kenya & G & 3 \\
\hline Lesotho & G & 3 \\
\hline Liberia & G & 3 \\
\hline
\end{tabular}


Table 1 Continued

\begin{tabular}{lll}
\hline Country/region & State of epidemic & Quality rating 2009 \\
\hline Madagascar & C & 1 \\
Malawi & G & 3 \\
Mali & G & 3 \\
Mauritania & C & 1 \\
Mauritius & C & 2 \\
Mozambique & G & 2 \\
Namibia & G & 2 \\
Niger & G & 2 \\
Nigeria & G & 3 \\
Rwanda & G & 3 \\
Sao Tome & G & 2 \\
Senegal & C & 3 \\
Sierra Leone & G & 2 \\
Somalia & C & 1 \\
South Africa & G & 3 \\
Swaziland & G & 2 \\
Togo & G & 3 \\
Uganda & G & 3 \\
United Republic of Tanzania & G & 3 \\
Zambia & G & 3 \\
Zimbabwe & G & 3 \\
\hline G conceris & &
\end{tabular}

C, concentrated; G, generalised; L, low level; 1, not enough information; 2, partially implemented; 3, good.

Dominican Republic, Haiti and India in 2006. These surveys were applied to adjust national HIV and global HIV estimates in recent estimation rounds.

Table 4 presents the regions and countries that have conducted national population-based surveys with the level of HIV prevalence. The prevalence ranges from $0.3 \%(0.23-0.33)$ in India in 2006 to $25 \%$ in Botswana in 2008.

\section{LOW AND CONCENTRATED EPIDEMICS}

\section{South and Southeast Asia (excluding China and India)}

Among the 18 countries assessed in this region, six are characterised as having fully functioning surveillance systems (Myanmar, Thailand, Cambodia, Vietnam, Bangladesh and Nepal) and seven as partially functional, and five were rated as being of poor quality. These results are similar to the findings of the 2007 round. As in the 2007 assessment, the region as a whole has sufficient data on IDU and CSW populations, and information on MSM populations has only recently become available in some countries such as Myanmar, Thailand and Cambodia. Despite these recent efforts, only a few countries can provide trends among these population groups.

\section{CHINA}

The Chinese surveillance systems and analysis of data have continued to improve over the last few years. There are considerably more studies in the international literature reporting results of HIV surveys and implications for policies ${ }^{8}$ but no additional research was done besides the epidemiological surveillance reports published. In 2007 and 2009, the national

Table 2 Comparison of final scores in 2007 and 2009

\begin{tabular}{lcc}
\hline Scores final & Scores 2007 & Scores 2009 \\
\hline Poorly functional & 53 & 56 \\
Partially functional & 45 & 47 \\
Fully functional & 40 & 35 \\
& 138 & 138 \\
\hline
\end{tabular}

government released estimates of provincial level HIV prevalence in the country using standard estimation methods. China continues to be rated as having a fully functioning surveillance system.

\section{INDIA}

The 2006 national population-based survey has contributed to the revision of national estimates since 2007, thus having an impact on the overall and global HIV estimates. ${ }^{9}$ Sentinel surveillance systems have continued to expand. During 2007, HIV Sentinel Surveillance was conducted at 1134 sites across the country. In 176 districts with 484 sites for ANC, 137 sites for female sex workers (FSWs) and 52 sites for IDUs, 40 for MSM and 248 for sexually transmitted disease clinics. ${ }^{10} 11$ There is also new information on HIV prevalence among MSM, and a recent evaluation of the Avahan initiative presents new data and impact of interventions recently published. India also continues to be rated as having a fully functioning surveillance system. ${ }^{12}$

\section{EASTERN EUROPE AND CENTRAL ASIA}

The 23 countries in this region all continue to have low-level or concentrated epidemics. Of the countries in this region, 10 have developed at least partially functioning surveillance systems, and 16 have poorly functioning systems. Weaknesses in this region included: (1) relying solely on case reporting, (2) the absence of repeated surveillance activities consistent over time across sites and (3) relatively few studies among MSM to be able to produce trends. Ukraine and Russia have increased the number of surveys among different population groups with high-risk behaviours, but changes in methods and locations make it difficult to assess trends.

\section{LATIN AMERICA AND THE CARIBBEAN}

Of the 27 countries in this region, all but one are designated as low-level or concentrated, with Haiti classified as having a generalised epidemic. Five national surveillance systems were scored as fully functioning, 11 as partially functioning and 11 as poorly functioning. These results are very similar to those reported in 2007 .

\section{NORTH AFRICA AND THE MIDDLE EAST}

For the 20 countries in this region, all have low-level or concentrated epidemics, but only two countries in the region have substantial data on HIV prevalence in different population groups, Morocco and Sudan. Sudan has made good efforts to improve its HIV surveillance system in the last few years. There is a need to conduct behavioural studies in the region to determine which are the main behaviours placing individuals at risk for infection. There are a few studies among CSWs, and new data have revealed that HIV is present among MSM and IDUs. ${ }^{13}$ However, HIV and AIDS case-reporting systems are in place and provide basic information in the region. Considerable efforts have been made in this region, and more data are available, but the assessment of epidemic trends remains a challenge.

\section{DISCUSSION}

The quality of national HIV surveillance systems, using the same approach to score countries as that applied in 2007, continues to reflect considerable variation, within and across regions. In sub-Saharan Africa, where over $68 \%$ of the global number of HIV infections occurs, the surveillance systems continue to be of generally good quality, particularly in Southern 
Table 3 Comparison of country scores between 2007 and 2009 by subregion

\begin{tabular}{|c|c|c|c|c|c|c|c|c|c|c|c|c|}
\hline & \multicolumn{2}{|l|}{ Africa } & \multicolumn{2}{|c|}{ SSEAsia } & \multicolumn{2}{|l|}{ EECA } & \multicolumn{2}{|l|}{ LA } & \multicolumn{2}{|c|}{ Caribbean } & \multicolumn{2}{|c|}{ MENA } \\
\hline & $\overline{2007}$ & 2009 & 2007 & 2009 & 2007 & 2009 & 2007 & 2009 & 2007 & 2009 & 2007 & 2009 \\
\hline Poorly functional & 6 & 8 & 5 & 5 & 12 & 13 & 6 & 7 & 6 & 4 & 18 & 18 \\
\hline Partially functional & 14 & 15 & 7 & 7 & 10 & 10 & 10 & 10 & 2 & 4 & 2 & 2 \\
\hline Fully functional & 25 & 22 & 7 & 7 & 1 & 0 & 4 & 3 & 3 & 3 & 0 & 0 \\
\hline Total & 45 & 45 & 19 & 19 & 23 & 23 & 20 & 20 & 11 & 11 & 20 & 20 \\
\hline
\end{tabular}

SSEAsia, South East Asia; EECA, Eastern Europe and Central asia; LA, Latin America; MENA, Middle East and North Africa.

Africa, with the world's highest HIV prevalence. Moreover, the number of ANC sites has continued to grow, an increase largely attributable to new sites included in surveillance systems in South Africa. Eight countries have conducted two or even three national surveys. More HIV surveillance data in many countries have led to improved assumptions in the estimation methodology, and the HIV estimates in these countries have become more accurate over time. Furthermore, these countries estimate HIV incidence levels using modelling or alternative approaches, and results can be compared to assess the validity of findings. ${ }^{14}$

In several countries, HIV surveillance systems have deteriorated in the last 2 years and are not performing as well as they should. This is especially the case in Central Africa and in some countries in East and West Africa that have been involved in recent political unrest.

High-quality surveillance systems are found in South and Southeast Asia. Like sub-Saharan Africa, the epidemics in some countries in this region are older than the rest of the world, and surveillance systems in countries with mature epidemics are generally functioning well. Only about a third of the surveillance systems in Latin America and the Caribbean have sufficient information to qualify as fully functioning surveillance systems. In the other regions (North Africa and the Middle East, and Eastern Europe and Central Asia), surveillance systems are either partially functioning or functioning poorly.

The results of this assessment reveal weaknesses of HIV surveillance systems in too many (56 of 138) of the evaluated countries, especially in countries with a low level of HIV infection or where the epidemic is concentrated in certain population groups. The number of such countries with studies considering MSM has increased overall, and there are now more data available on this population in many regions, including Africa. Because of a lack of consistency in surveillance efforts, it is very difficult to assess trends, but substantial progress has been made in studying the epidemic within this group in the last few years. Regarding FSWs, the number of studies has decreased in recent years, and in most countries no trend data are available.

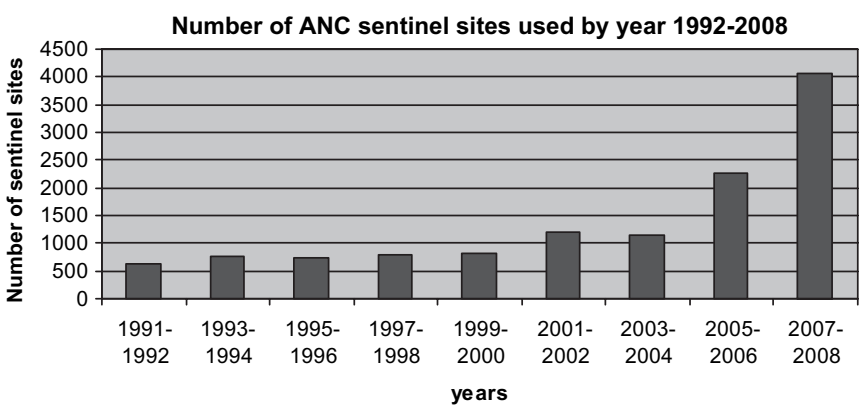

Figure 1 Number of sites included in antenatal clinic surveillance in sub-Saharan Africa, 1992-2008.
In countries in Central and Eastern Europe, most of the epidemiological data available are on injecting-drug users, but even in these countries trends in the epidemic are difficult to monitor due to the lack of systematic methods to undertake surveillance studies.

When compared with 2007 results, 35 countries have fully functioning systems in 2007 versus 40 in 2009, and 45 have

Table 4 Adult (aged 15-49) national population-based surveys that included HIV testing, 2001-2008

\begin{tabular}{ll}
\hline Country/region & Year \\
\hline Asia & \\
Cambodia & 2005 \\
India & $2005-2006$ \\
Papua province (Indonesia) & 2006 \\
Hai Phong province (Viet Nam) & 2005 \\
Caribbean & 2002 \\
Dominican Republic & 2007 \\
Haiti & 2004 \\
Sub-Saharan Africa & \\
Benin & 2006 \\
Botswana & 2004,2008 \\
Burkina Faso & 2003 \\
Burundi & 2002 \\
& 2007 \\
Cameroon & 2004 \\
Central African Republic & 2006 \\
Chad & 2005 \\
Congo & 2009 \\
Côte d'Ivoire & 2005 \\
Democratic Republic of the Congo & 2007 \\
Djibouti & 2002 \\
Equatorial Guinea & 2004 \\
Ethiopia & 2005 \\
Ghana & $2005-2006$ \\
Guinea & \\
Kenya & 2003 \\
Lesotho & 2005 \\
Liberia & $2003,2007,2009$ \\
Malawi & 2004 \\
Mali & 2007 \\
Niger & 2004 \\
Nigeria & 2001,2006 \\
Rwanda & 2002 \\
Senegal & 2006 \\
Sierra Leone & 2007 \\
South Africa & 2005 \\
Swaziland & 2005 \\
Uganda & 2008 \\
United Republic of Tanzania & 2005 \\
Zambia & $2002,2005,2008$ \\
\hline & $2006-2007$ \\
Zimbabwe & 2005 \\
& 2007 \\
& \\
& \\
& \\
&
\end{tabular}


partially functioning systems in 2007 versus 47 in 2009. Poorly functioning systems increased from 53 in 2007 to 56 in 2009. Even though there is a small increase on number of countries with not functioning systems, those countries represent a small proportion of the global burden of disease. Therefore the great proportion of the new global HIV estimates are based in countries with good surveillance systems.

The countries in North Africa and Middle East, with a few exceptions, continue to have limited HIV surveillance activities. While there are now more data available than before, trends in the epidemic can still not be assessed, and the countries in this region therefore received low scores.

One of the reasons why countries remain in either score with poor or partially systems is mostly because of the lack of consistency of methods and tools. Even though some countries have increased the number of surveys and have more data in different population groups, the instruments and tools used for survey are different. Most of the countries are using Respondent Driving Sampling (RDS) as a strategy for sampling on high-risk groups. However, in the past, other approaches such as venue sampling or time location sampling have been used, so it is not possible to construct trends.

This paper considered data available for the last 9 years to assess the quality of surveillance systems that are being used for monitoring the HIV epidemic. In countries with the greatest disease burden, surveillance has continued to improve over time, and the addition of large population-based HIV prevalence surveys in these countries has greatly enhanced the reliability of the data. In many other countries, specifically those with low-level and concentrated epidemics, the quality of data has remained very similar, although many countries still lack the consistency required to follow trends over time in high-risk populations. There are gaps in some country data on high-risk populations, and behavioural data are generally scarce.

The parameters used to score surveillance systems are not perfect, as scoring is based on public reports. Because providing trends is one of main properties of sentinel surveillance, the frequency of surveys has more weight than the other parameters. The longer the time assessed, the more weight this parameter carries. Recent data on the timeliness of information is another important parameter as it assesses whether surveillance data are recent or not, and it has implications for the estimates produced at the country level. ${ }^{15}$ In spite of the limitation on the scoring system, similar parameters and weights were kept to compare them with the 2007 assessment of quality.

\section{Limitations}

There are several limitations in this analysis. First, we may have not access to all the surveillance reports and studies conducted in countries that remain in the grey literature. Second, the assessment criteria may be unfair to small countries with low epidemic levels. Those countries may have opted for surveillance systems based mostly on case reporting, rather than surveys. In these local communities, integrated behaviour surveys among populations with high-risk groups may be very difficult to undertake. Lastly the score system is not perfect, as intends in assessing HIV trends and coverage of HIV surveillance among different populations. It does not address other issues as qualitative surveys or the use of data.

Every effort has been made to contact regional experts and national epidemiologists via WHO regional offices and to ensure that most recent surveillance data have been identified for this

\section{Key messages}

The quality of HIV surveillance systems remains at the same level as that for 2007 in high-burden countries.

- More national population surveys have been conducted worldwide, and these provide better coverage in generalised epidemics.

- There is a need to review and evaluate HIV surveillance systems on a regular basis.

- A significant number of countries still need to improve their HIV surveillance systems.

study. Appropriate data for the assessment of surveillance quality in low-level or concentrated epidemics were limited to high-risk populations, while the analysis in countries with generalised epidemics was based on antenatal clinic surveillance and population-based surveys that include HIV testing. Other sources of data that are used in countries for monitoring the epidemic, such as blood screening mechanisms, were not captured here. Publications in languages other than English, French, Spanish or Russian were not considered in this study.

Several recommendations can be made based on our analysis. First, there is a need to review and assess HIV surveillance system periodically, to see if HIV surveillance systems are responding to the needs of the countries. ${ }^{15}$ These reviews or evaluations can be made regularly by national experts, and occasionally by external experts. Second, the consistency of methods, tools used, populations and geographical locations are the key parameters for surveillance to detect trends and overall burden of disease. Third, resources should be made available to improve the quality data collected.

Acknowledgements The authors would like to thank all national epidemiologists and international staff, and E Fadriquela for their assistance in the management of the data associated with this study.

\section{Competing interests None.}

Contributors JMGC drafted the first draft. All the other authors contributed to data collection from the various countries and scoring systems, and provided comments and ideas to the first draft.

Provenance and peer review Not commissioned; externally peer reviewed.

\section{REFERENCES}

1. UNAIDS/WHO. Guidelines For Second Generation HIV Surveillance. UNAIDS/WHO Working Group on global HIVIAIDS and STI Surveillance. Geneva: UNAIDS/WHO, 2000.

2. Walker N, Garcia-Calleja JM, Heaton L, et al. Epidemiological analysis of the quality of HIV sero-surveillance in the world: how well do we track the epidemic? AIDS 2001:15:1545-54.

3. Garcia-Calleja JM, Zaniewski E, Ghys PD, et al. A global analysis of trends in the quality of HIV sero-surveillance. Sex Transm Infect 2004;80(Suppl 1):i25-30.

4. Lyerla R, Gouws E, Garcia-Calleja JM. The quality of sero-surveillance in low- and middle-income countries: status and trends through 2007. Sex Transm Inf 2008:84:185-91.

5. UNAIDS/WHO EpiUpdate 2009. Joint United Nations Programme on HIVIAIDS (UNAIDS). Geneva, Switzerland: UNAIDS/WHO, 2009

6. Department of Health. Ministry of Health, South Africa, 2008 HIV Surveillance and Syphilis Among Pregnant Women. Pretoria, South Africa, 2008.

7. WHO. Sentinel Surveillance for HIV infection. Geneva: WHO/GPA/DIR/88.8, 1988

8. Wu Z, Wang Y. China meets new AIDS challenges. J Acquir Immune Defic Syndr 2010;53(Suppl 1)

9. National AIDS Control Program. HIV Sentine/ Surveillance and HIV Estimation 2007-A Technical Brief. New Delhi, India: Ministry of Health, 2007.

10. National AIDS Control Program. India Operational Guidelines for HIV Sentinel Surveillance. New Delhi, India: Ministry of Health, 2007

11. National AIDS Control Program. Operational Guidelines for HIV Sentinel Surveillance Round. New Delhi, India: Ministry of Health, 2008. 


\section{Supplement}

12. Chandrasekaran P, Dallabetta G, Loo V, et al. Evaluation design for large-scale HIV prevention programmes: the case of Avahan, the India AIDS initiative. AIDS 2008;22:S1-S15.

13. World Health Organization/EMRO Office. HIVIAIDS Epidemic Situation in the Eastern Mediterranean Region. Cairo: WHO Eastern Mediterranean Region Office, 2009. In press.
14. Andrea K, Hallett T, Stover J, et al. Measurement of HIV incidence among adults in Kenya and Uganda: a systematic comparison of multiple method paper 992 CROI (Conference of Retrovirus and Opportunistic Infections) 2010.

15. Stover J, Johnson P, Zaba B, et al. The Spectrum projection package: improvements in estimating mortality, ART needs, PMTCT impact and uncertainty bounds. Sex Transm Infect 2008;84(suppl 1):i24-30. 